\title{
Acute Hepatitis-Like Syndrome Revealing Hashimoto Thyroiditis
}

\author{
Salem Bouomrani*, Mouna Guermazi, Souad Yahyaoui and Hanene Nouma \\ Department of Internal Medicine, Military Hospital of Gabes, Tunisia
}

*Corresponding author: Salem Bouomrani, Department of Internal medicine, Military Hospital of Gabes, Tunisia.

Received Date: January 12, 2019

Published Date: January 22, 2019

\begin{abstract}
Introduction: Disturbances of hepatic biologic tests are not uncommon during hypothyroidism but classically refer to rhabdomyolysis. More rarely, moderate and reversible chronic cholestasis or hypertransamenaemia can be observed. Acute hepatitis-like syndrome remains exceptional.

Case report: 30-year-old patient with no pathological medical history was referred to us by her family doctor for suspicion of acute viral hepatitis: ASAT at $4 \mathrm{~N}$ and ALAT at 3N, asthenia, abdominal pain, and vomiting evolving for three days in an epidemic context of hepatitis A. The clinical examination noted bradycardia at 58/min and macroglossia. Hepatic tests confirmed hepatocellular necrosis without cholestasis or hepatocellular insufficiency: ASAT at 355IU/l (8N), ALAT at 243IU/l (6N), total bilirubin at 14.80 $\mu$ mol/l, GGT at 15IU/l and PT at 100\%. The serodiagnostics of viral hepatitis A, B and C, and specific liver autoantibodies were negative. Thyroid assessment revealed deep primary hypothyroidism with TSH at $182.7 \mu \mathrm{mol} / \mathrm{ml}$. The evolution under hormonal substitution treatment was favorable with disappearance of the clinical complaints and normalization of the liver tests with the normalization of the TSH.

Conclusion: Acute hepatocellular necrosis remains an exceptional presentation of hypothyroidism. His diagnosis is not always easy, especially since in the autoimmune context of Hashimoto's thyroiditis, the association with true autoimmune hepatitis can be discussed. As well as a thyroid disorder associated with chronic viral hepatitis. A screening of the thyroid function is thus justified in front of any hepatocellular necrosis that is not proven.
\end{abstract}

Keywords: Acute hepatitis, Hypothyroidism, Hashimoto thyroiditis, Hepatitis-like syndrome, Hypertransaminasemia

\section{Introduction}

Described for the first time in 1912 by the Japanese doctor Hakaru Hashimoto [1], the thyroiditis that had been bearing his name is currently considered to be the most common autoimmune disease in the world [2] and represents a major health problem [3]. Its prevalence in the United States of America is estimated at 4-13\% of the general population [3].

This endocrinopathy is characterized by a large clinical polymorphism making its diagnosis a real challenge for clinicians [4-5]. Indeed, its spectrum of clinical presentations can range from completely asymptomatic forms (silent thyroiditis) to the most severe forms of Hashimoto encephalopathy, via subclinical forms and "unusual" or "atypical" forms of presentation [5-8].

Disturbances in hepatic biologic tests are not uncommon during hypothyroidism, but classically refer to rhabdomyolysis [9]. More rarely, moderate chronic cholestasis and, exceptionally, isolated hypertransamenaemia can be observed which are reversible [9-10]. Pseudo-acute hepatitis syndrome (acute hepatitis-like syndrome) remains an unusual presentation of this autoimmune endocrinopathy [11-12].

We report an original observation of isolated hypertyrosinemia simulating acute hepatitis as an inaugural presentation of Hashimoto's thyroiditis (HT).

\section{Case Report}

30-year-old patient with no pathological medical history was referred to us by her family doctor for suspicion of acute hepatitis: hypertransaminasemia with ASAT at $177 \mathrm{IU} / \mathrm{l}(4 \mathrm{~N})$ and ALAT at $122 \mathrm{IU} / \mathrm{l}(3 \mathrm{~N})$, asthenia, abdominal pain, and vomiting evolving for three days in an epidemic context of hepatitis A. 
The clinical examination at admission found an aporetic patient, conscious, and well oriented without jaundice or other progressive skin lesions. We particularly noted a bradycardia at 58/min and macroglossia (Figure 1) with bilateral dental impressions (Figure 2).

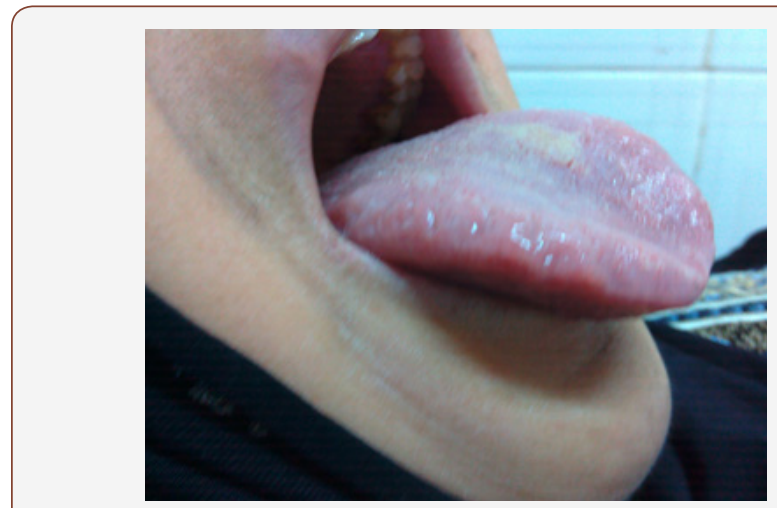

Figure 1: Macroglossia

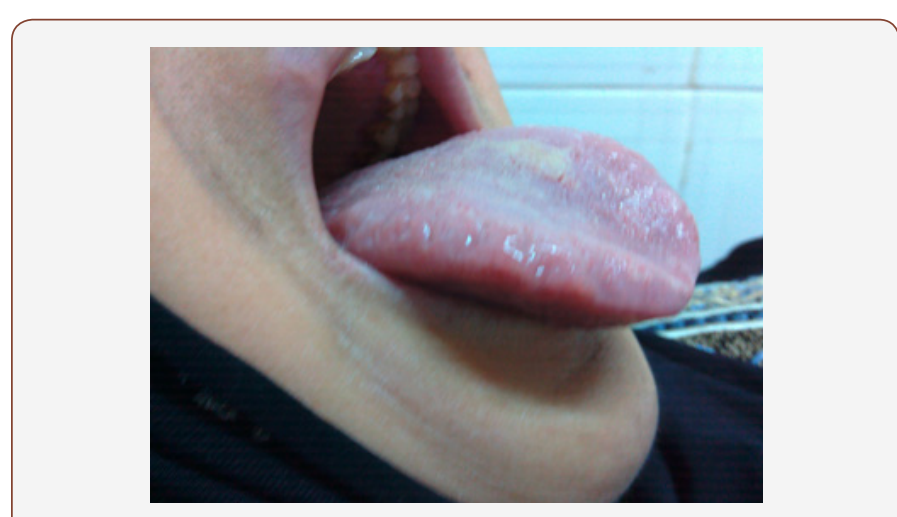

Figure 2: Dental impressions on the right-side edge of the tongue.

The biology confirmed the diagnosis of isolated hepatocellular necrosis without cholestasis or hepatocellular insufficiency: ASAT at $355 \mathrm{IU} / \mathrm{l}(8 \mathrm{~N})$, ALAT at $243 \mathrm{IU} / \mathrm{l}(6 \mathrm{~N})$, total bilirubin at 14.80 $\mu \mathrm{mol} / \mathrm{l}, \mathrm{GGT}$ at $15 \mathrm{IU} / \mathrm{l}$, and PT at $100 \%$. There was also hypochromic microcytic anemia at $11 \mathrm{~g} / \mathrm{dl}$ and hypercholesterolemia at 7.30 $\mathrm{mmol} / \mathrm{l}$. The other biologic parameters were within normal limits: leukocytes, platelets, erythrocyte sedimentation rate, C-reactive protein, calcemic, creatinine, monogram, CPK and LDH.

Abdominal ultrasound showed no signs of acute or chronic liver disease. For this hepatitis, specific investigations were negative: serologies of viral hepatitis A, B and C, electrophoresis of serum proteins, anti-nuclear antibodies, anti-LKM1 antibodies, anti-M2 antibodies, anti-smooth muscle antibodies, ferritin, cupremia, and ceruloplasmin specifically eliminating viral hepatitis, autoimmune hepatitis, hemochromatosis or Wilson's disease.

The thyroid tests required for suspicion of hypothyroidism (macroglossia, bradycardia, hypercholesterolemia, and anemia) revealed profound primary hypothyroidism with a TSH at $182.7 \mu \mathrm{mol} / \mathrm{ml}$ and an fT $4<1 \mathrm{pmol} / \mathrm{l}$. Cervical ultrasound and the study of thyroid immunity confirmed the diagnosis of Hashimoto's thyroiditis: positive anti-thyroglobulin antibodies at $255.4 \mathrm{IU} / \mathrm{ml}$ and strongly positive anti-TPO antibodies at $1000 \mathrm{IU} / \mathrm{ml}$.
Thus the diagnosis of hypothyroidism by Hashimoto autoimmune thyroiditis was retained. Disruption of liver tests with the clinical presentation of acute hepatitis-like syndrome was related to hypothyroidism given the negativity of specific explorations.

Evolution under hormone replacement therapy was favorable with the disappearance of clinical complaints and normalization of liver tests with the return to normal of the TSH.

\section{Discussion}

Despite the good knowledge of the effect of thyroid hormones on the entire human metabolism, the effects of hypothyroidism on the liver are not always well understood [13]. However, minim liver injuries and/or minim to moderate abnormalities of hepatic enzymes are often frequently associated to thyroid diseases $[14,15]$.

On the other hand, disturbances of thyroid function, particularly with hypothyroidism, may be associated with chronic viral or autoimmune hepatitis $[16,17]$, thus making the diagnosis of liver test abnormalities concomitant with thyroid disorders a real diagnostic challenge.

Except for forms associated with rhabdomyolysis, hypertransaminasemias are rare in hypothyroidism and it is currently well established that hypothyroidism is only exceptionally responsible for isolated hypertyrosinemia [18]. In fact, most cases of hypertransaminasemia caused by hypothyroidism are reported as sporadic observations [9,11,12,19,20]. These hypertransaminasemias may be isolated or may be associated with cholestasis defining mixed hepatic injury [13] or more rarely integrate into the setting of more severe clinical presentation with hyperammonemia [21] or severe hepatic insufficiency with hepatic encephalopathy [22].

Hypertransaminasemia may exceptionally be the revealing sign of both acquired (adult) [20] and congenital (newborn) hypothyroidism [23] and no significant correlation was found between the importance of these enzymatic disturbances and the value of TSH [24].

Major acute hepatocellular necrosis remains an uncommon presentation of hypothyroidism: a single patient in Bensalem W et al series of 70 cases of hypothyroidism of the elderly (1.4\%) [10] and to our knowledge only two cases of acute hepatitis-like syndrome revealing hypothyroidism have been reported previously in the world literature [11-12] our observation is thus the third reporting this mode of revelation.

Disturbances of liver tests caused by hypothyroidism usually resolve rapidly after correction of thyroid hormone status [13,2124].

\section{Conclusion}

As exceptional as it is, this severe presentation of hypothyroidism deserves to be known and a screening of the thyroid function is thus justified in front of any unexplained hepatocellular necrosis. Our observation is, to our knowledge the third reporting Hashimoto thyroiditis revealed by an acute hepatitis-like syndrome. 
Abbreviations: ALAT: alanine-aminotransferase, ALP: alkaline phosphatase, Anti-LKM-1 antibodies: anti-Liver kidney microsome type 1 antibodies, anti-M2: anti-mitochondrial M2 antibodies, ASAT: aspartate-aminotransferase, CPK: creatine phosphokinase, GGT: gamma-glutamyl transpeptidase, LDH: lactate dehydrogenase, PT: prothrombin time, TSH: thyroid stimulating hormone.

\section{Acknowledgement}

None.

\section{Conflict of Interest}

No Conflict of Interest.

\section{References}

1. Hashimoto H, Zur Kenntniss der, lymphomatösen Veränderung der Schilddrüse (1912) Struma lymphomatosa. Arch Klin Chir 97: 219-248.

2. Caturegli P, De Remigis A, Rose NR (2014) Hashimoto thyroiditis: clinical and diagnostic criteria. Autoimmun Rev 13(4-5): 391-397.

3. Siegmann EM, Müller HHO, Luecke C, Philipsen A, Kornhuber J (2018) Association of depression and anxiety disorders with autoimmune thyroiditis: A Systematic review and meta-analysis. JAMA Psychiatry 75(6): 577-584.

4. Hernández Ramírez DA, Castellanos Juárez JC, Romero T, Barragan Rincón Á, Blanco Benavides R (2008) Mixedematous ileus; acute abdomen exacerbate. Rev Gastroenterol Mex 73(4): 231-234.

5. Chaker L, Bianco AC, Jonklaas J, Peeters RP (2017) Hypothyroidism. Lancet 390(10101): 1550-1562.

6. Bouomrani S, Regaïeg N, Ben Hamad M, Lassoued N, Belgacem N, et al. (2018) An Unexpected etiology of Rhizomelic Pseudo-Polyarthritis (Polymyalgia Rheumatica) in the elderly. Archives of Orthopedics and Rheumatology 1(1): 12-16.

7. Bouomrani S, Lassoued N, Ben Hamad M, Regaïeg N, Belgacem N, et al. (2018) Recurrent intestinal obstruction revealing hypothyroidism. Archives of Gastroenterology and Hepatology 1(1): 22-25.

8. Bouomrani S, Regaieg N, Belgacem N, Ben Hamed M, Lassoued N, et al. (2018) Myositis-like syndrome revealing hypothyroidism. Archives of Diabetes and Endocrine System 1(2): 1-3.

9. Nicolas X, Cholet F, Hiar I, Tilly Gentric A, Pennec YL (2000) Hypertransaminasemia and chronic cholestasis, adrenal insufficiency, hypothyroidism. Gastroenterol Clin Biol 24(10): 967-969.
10. Bensalem W, Lamloum M, Tougorti M, Bensalem T, Benghorbel I, et al. (2015) Hypothyroïdie du sujet âgé. Ann Endocrinol (Paris) 76(4): 451.

11. Conde Martel A, Aguilar Utrilla P, Artiles Vizcaíano J (1998) Vomiting and hypertransaminesemia as presenting form of hypothyroidism. Rev Esp Enferm Dig 90(10): 728-729.

12. Koklu E, Kurtoglu S, Akçakus M, Gunes T, Öztürk MA, et al. (2007) Cholestatic hepatitis like syndrome and congenital hypothyroidism. Erciyes Medical Journal 29: 168-171.

13. Duong N, Lee A, Lewis J (2018) Case of acute mixed liver injury due to hypothyroidism. BMJ Case Rep 2017-222373.

14. Huang MJ, Liaw YF (1995) Clinical associations between thyroid and liver diseases. J Gastroenterol Hepatol 10(3): 344-350.

15. Malik R, Hodgson H (2002) The relationship between the thyroid gland and the liver. QJM 95(9): 559-569.

16. Shen Y, Wang XL, Xie JP, Shao JG, Lu YH, et al. (2016) Thyroid disturbance in patients with chronic hepatitis $\mathrm{C}$ infection: A systematic review and meta-analysis. J Gastrointestin Liver Dis 25(2): 227-234.

17. Guzman Rojas P, Gallegos Lopez R, Ciliotta Chehade A, Scavino Y, Morales A, et al. (2016) Autoimmune hepatitis induced by isotretionine. Rev Gastroenterol Peru 36(1): 86-89.

18. Clarke EA, Yates P, Goldie DJ (1989) Hypothyroidism: an important differential diagnosis for raised serum transaminases? J Clin Pathol 42(11): 1226-1227.

19. Serranti D, Indolfi G, Bartolini E, Galli L, de Martino M, et al. (2013) Raised serum aminotransferase levels and muscle pseudohypertrophy caused by hypothyroidism. J Pediatr Gastroenterol Nutr 56(6): 48-49.

20. De Diego Lorenzo A, Santos L, Catalina V, García Sánchez A, Escudero M, et al. (2001) Constipation and hypertransaminasemia as presentation form of hypothyroidism. Rev Esp Enferm Dig 93(4): 263-264.

21. De Nardo D, Franconi G, Sabino D (1999) Hyperammonemia during hypothyroidism: an unusual biohumoral finding normalized by hormonal replacement treatment. Ann Ital Med Int 14(3): 196-201.

22. Thobe N, Pilger P, Jones MP (2000) Primary hypothyroidism masquerading as hepatic encephalopathy: case report and review of the literature. Postgrad Med J 76(897): 424-426.

23. Mantri R, Bavdekar SB, Save SU (2016) Congenital hypothyroidism: An unusual combination of biochemical abnormalities. Case Rep Pediatr 2016: 2678578 .

24. Saha B, Maity C (2002) Alteration of serum enzymes in primary hypothyroidism. Clin Chem Lab Med 40(6): 609-611. 